\title{
Fibrocartilaginous Embolism of The Spinal Cord - A Case Report
}

\section{Ademar Lucas Junior}

Sao Camilo Diagnostic Medicine, Diagnostic Imaging, Santos Dumont St, Maringa, Parana, Brazil

Corresponding author: Ademar Lucas Junior, Neuroradiologist, Sao Camilo Diagnostic Medicine, Diagnostic Imaging, Santos Dumont St, Maringa, Parana, Brazil. Tel: 5511 992933038; E-mail: ademarlucasjr@gmail.com

Received: Sep 26, 2016; Accepted: Dec 27, 2016; Published: Dec 30, 2016

Citation: Junior AL. Fibrocartilaginous Embolism of The Spinal Cord - A Case Report. Jneurol Neurosci. 2016, 7:6.

\section{Abstract}

Fibrocartilaginous embolism of the spinal cord is considered a rare ischemic vascular syndrome [1-4], though some authors believe it to be underdiagnosed. It results in abrupt onset and rapidly progressive sensory, motor and autonomic neurologic deficits.

This paper reports a case of a teenage patient presenting with sudden onset rapidly progressive back pain associated with paraparesthesia, paraparesis and urinary retention after physical exertion. A magnetic resonance imaging (MRI) test led to a presumptive diagnosis of spinal cord fibrocartilaginous embolism.

Keywords: Fibrocartilaginous embolism; Spinal cord; Ischemic vascular syndrome; Autonomic; Neurologic deficits

\section{Introduction}

Fibrocartilaginous embolism of the spinal cord is considered a rare condition, with a poor and potentially fatal prognosis [1-4]. This entity is characterized by a sudden onset of clinical signs following a traumatic episode, with a peak of neurologic deficit in the first hours / days and usually normal CSF tests. Radiologically, it presents typical imaging findings (on MRI) that lead to the presumptive diagnosis of spinal cord ischemia caused by fibrocartilaginous emboli [5].

Its incidence is bimodal, affecting both adolescents and older adults $[1,3,5]$ due to the characteristics of spinal cord vascularization in each of these age groups $[3,5]$.

Low-energy traumas can cause rupture of the annulus fibrosus, with subsequent displacement of emboli from the nucleus pulposus of intervertebral discs to the inside of the arterial branches that supply the spinal cord parenchyma, causing arterial occlusion and subsequent spinal cord ischemia $[1-3,5]$.

\section{Case Report}

A 14-year-old male patient, otherwise healthy, presenting with sudden onset back pain after physical effort (attempt to lift the sofa), evolving with paraparesthesia with no sensory level, and paraparesis (grade 3 and grade 4 strength on the left and right sides, respectively) within hours. He was taken to the emergency room, where laboratory tests (blood count, urinalysis, CSF) were ran and showed no abnormalities. During his hospital stay, he evolved with urinary retention, for which a urinary catheter was placed.

The patient was then sent to the imaging center for an MRI study, which showed major signal abnormalities in the thoracic segment of the spinal cord (between D2 and D9), with hyperintense signal on the axial view of the T2 weighted sequence, affecting the anterior two thirds of the spinal cord. There was no abnormal enhancement response to the contrast medium (Figure 1). A complementary echo-planar diffusionweighted MRI was conducted and revealed intense restriction on the free movement of water molecules, matching the ADC map findings (Figure 2). It also showed multiple Schmorl's hernias, edema and enhanced vertebral plateaus (acute herniation) coinciding with the segment of the spinal cord findings.

The patient stayed in hospital for a week, with partial improvement of his neurological status, complete recovery of the autonomic / bladder function, partial recovery of lower limbs strength (grade 4 bilaterally), and no other deficits.

\section{Discussion}

Fibrocartilaginous embolism is a rare clinical entity, having only a few cases reported in the literature $[1,2,5]$. Little is known about the condition, and disseminating knowledge about it is important so as to improve diagnostic accuracy and make it possible for specific therapies to be developed $[1,6]$.

The diagnosis of a high percentage of cases is only achieved post mortem $[1,2]$. In spite of that, clinical signs supported by imaging tests (MRI) can lead to the correct presumptive diagnosis $[1,6]$.

The first case was reported in 1961 by Naiman et al. $[1,3,5,7]$. After that, only a few cases were reported in the literature and most of those patients died of neurologic complications [1-5]. 

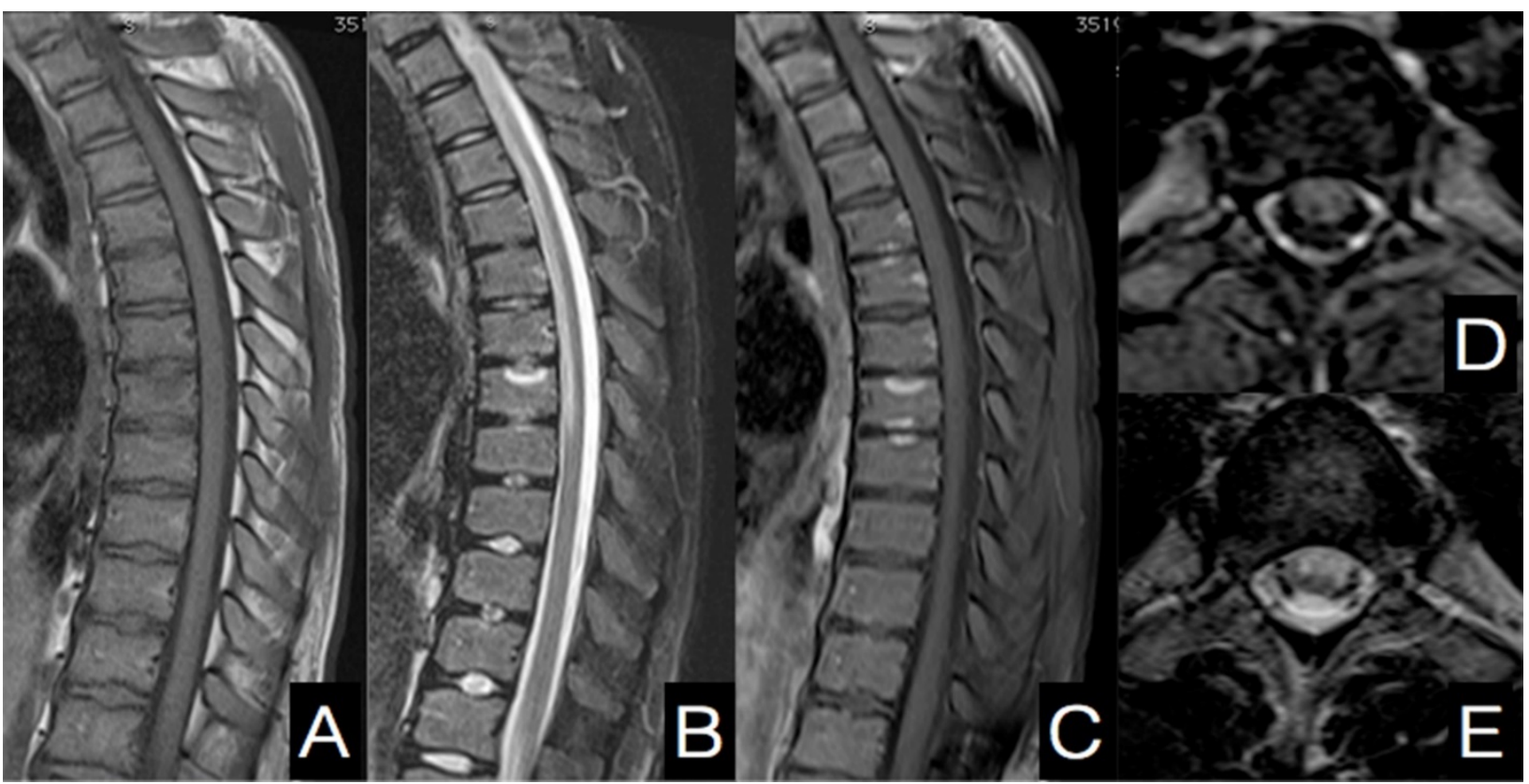

Figure $1 \mathrm{MRI}$ sequences: sagittal view (A: pre-contrast T1 FSE, B: T2 FSE, and C: post-contrast T1 FSE); and axial view (D: postcontrast T1 FSE, and E: T FSE). There are signal abnormalities in the superior thoracic spinal cord parenchyma extending longitudinally over more than three vertebral bodies, and involving the anterior two thirds of the spinal cord parenchyma (E), characterized as hyperintense signal on the T2 weighted sequence (myeloedema). There is no spinal cord enhancement response to the paramagnetic contrast $(C$ and $D)$. The sagittal view images also reveal acute intrasomatic herniations (Schmorl) through the superior and middle thoracic vertebral plateaus, which correspond to the level of the spinal cord findings.

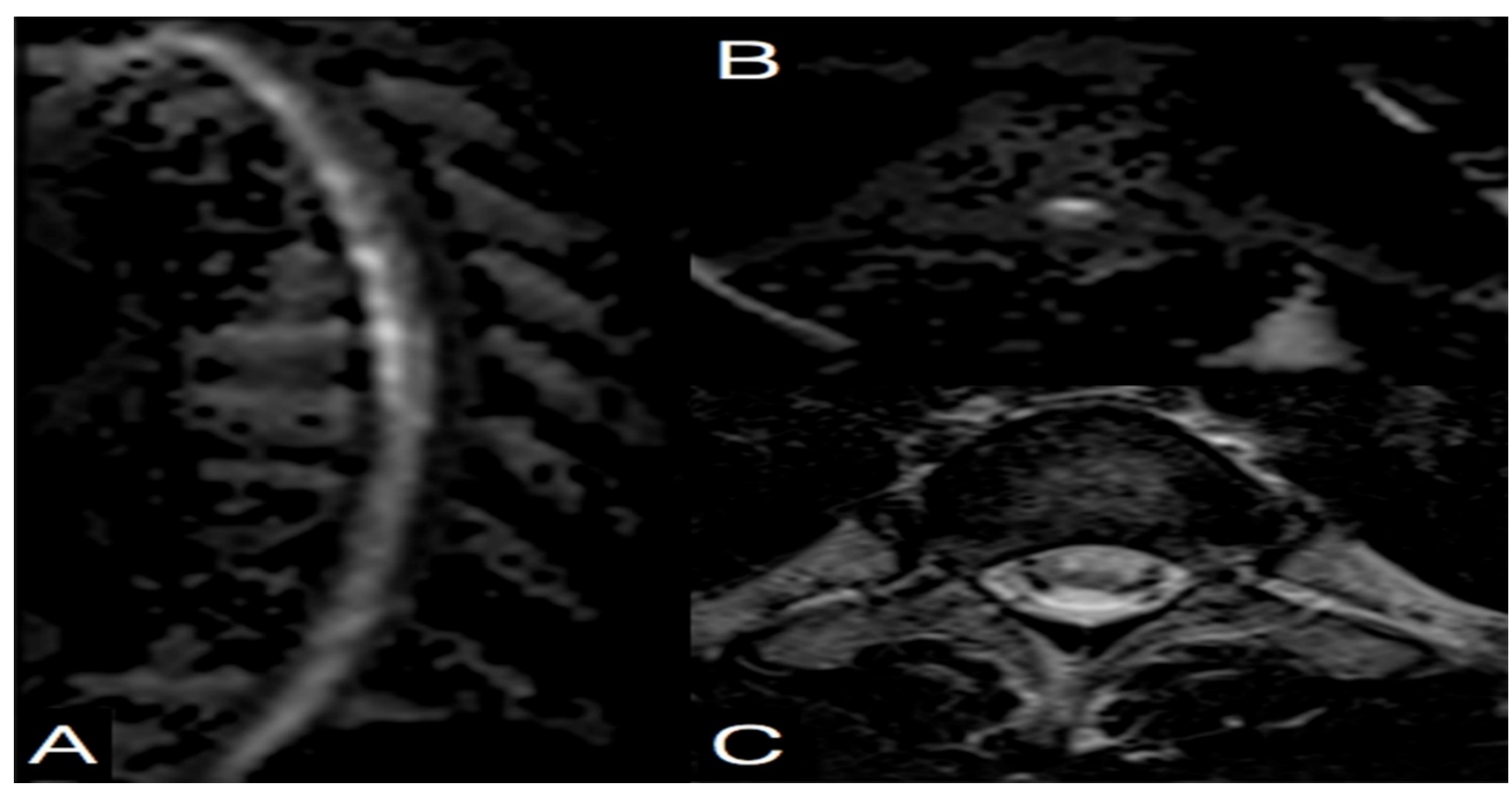

Figure 2 Signal abnormalities in the spinal cord parenchyma are seen as hyperintense signal on the echo-planar diffusionweighted sequence ( $A$ and $B$ ), compatible with cytotoxic edema (acute spinal cord ischemia).

This clinical entity is believed to be underdiagnosed [1,3], especially when it comes to the incidence of non-fatal cases [3]. False positives are also possible as there are similar neurologic syndromes, such as acute transverse myelitis, anterior spinal artery syndrome [1,3], and Guillain Barré syndrome [1]. 
In fatal cases, the cervical spine seems to be the most commonly affected [1,5], which suggests that the spinal cord damage may extend to the bulbomedullary transition, with a consequent impairment of vital functions (cardiorespiratory system).

It is slightly more predominant in females $[1,2,5,6]$ and has a bimodal incidence, with a peak among adolescents/ young adults (on average 22 years of age) and another one among older adults (60 years of age, on average) $[1,3,5]$.

A classically described trigger is trauma, which can vary from low- to high-energy (ranging from falls to car accidents) $[1-3,6]$, but there are also other possible triggering factors like pregnancy, use of oral contraceptive pills or corticoisteroids [1].

The intervertebral discs are the largest avascular structure in the human body, and their nutrition in adults occurs by diffusion [8]. During childhood, the intervertebral discs receive nutrients from the same source that irrigates the spinal cord, which subsides in adolescence. The persistence of this type of vascularization is reported by some authors as the cause of the higher incidence of fibrocartilaginous embolism among adolescents $[3,5]$. Similarly, the emergence of neovascularization may be related to the higher incidence in older adults $[3,5]$.

The spinal cord is supplied by: 1) the anterior spinal artery, which is a branch of the vertebral arteries and supplies the anterior two thirds of the spinal cord; 2) two posterior spinal arteries, which are branches of the posteroinferiores cerebellar arteries, responsible for the blood supply of the posterior third of the spinal cord; and 3) the radicular arteries among which one must highlight the arteria radicularis magna (Adamkiewicz), a branch of the aorta and the main source of T8 to $L 1$ vascularization $[4,9]$.

When both the anterior and posterior spinal arteries are involved, the patient presents motor and sensory deficit [4], since the anterolateral portion of the cord is responsible for thermal, tactile and pain sensitivity, whereas the posterior portion is responsible for proprioception and vibration. When the arteria radicularis magna is affected the patient progresses to fecal and urinary incontinence, as well as motor deficit [9].

The venous system of the spinal cord is divided into intrinsic-composed of radial and sulcal veins-and extrinsic, composed of the anterior and posterior spinal veins. The latter corresponds to the anterior and posterior vertebral arteries and drains to the radiculomedullary veins, which in turn communicate with the epidural venous system [9].

As to the venous drainage of the vertebrae, it is performed by the basivertebral veins coming from inside the vertebral body and draining to the external vertebral venous plexus [9].

The pathophysiology of this type of injury is characterized by the presence of fibrocartilage (from the intervertebral disc) in the medullary vessels, blocking them and resulting in spinal cord ischemia. This fibrocartilaginous material usually stems from the rupture of the annulus fibrosus and may be associated with the presence of acute Schmorl's nodes $[1,2,8]$.
Debris of fibrocartilage (from the nucleus pulposus) can be drained from the sinusoids to the basivertebral vein and then to the epidural plexuses due to an increase in blood pressure. Such increase in the pressure of perivertebral vessels causes a retrograde blood flow that takes potential emboli to the cord [1-4].

There are also reports of increased intradiscal pressure related to an axial overload that could push emboli from the sinusoids to the spinal cord [3].

This causes spinal cord infarction with sudden and rapidly progressive neurologic deficit, preceded by complaints of sudden neck or back pain $[1,3-5,10,11$,$] . The neurologic$ symptoms will depend on the level of the injury and its extent, ranging from loss of limb strength to paraplegia [9]. There are reports stating that the onset of paraplegia may occur within hours to about 2 to 3 days after the first pain complaints $[5,9]$.

Pain is described in approximately $70 \%$ of patients with acute spinal cord ischemia and usually occurs at the level of the embolism [9] and this finding in association with previous history of trauma may be an important clinical data in diagnosis of this entity to the detriment of their differentials.

Histopathological diagnosis requires the characterization of the embolus in the spinal cord vasculature, which makes it unfeasible in clinical practice [1], hence the importance of imaging tests to identify any medullary abnormalities.

$\mathrm{MRI}$ is the most sensitive test to detect spinal cord ischemia $[2,3,9]$. This condition is characterized by hyperintensity on $\mathrm{T} 2$ $[2,9]$, increased spinal cord size during the acute/early subacute phase, with variable enhancement response to the intravenous contrast medium, being highly dependent on the evolution of the condition [2,9]. Areas of hemorrhage and, later on, medullary necrosis can be seen [2]. There are reports of patients with an initial normal MRI [3], however the diffusion-weighted sequence can increase the sensitivity and specificity of the diagnosis in acute cases [4,9], with hyperintense signals matching the $A D C$ map findings. If the patient does not undergo any specific therapy and there is no spontaneous recanalization, the cytotoxic edema progresses to irreversible spinal cord damage with atrophy of the involved spinal segments, which indicates myelomalacia [5].

As for the lab tests, there are reports of an incipient increase in CSF protein levels in some patients [2].

Among the differential diagnoses are the other causes of spinal cord ischemia/ infarction, such as prolonged arterial hypotension, disc herniation, demyelinating diseases, and aortic thrombo-occlusive disease [2,3].

Regarding the treatment of medullary fibrocartilaginous embolism, there is no specific guideline to date [6]. There are reports of use of anticoagulation, intravenous steroids and immunoglobulins, as well as plasma exchange, but all these without significant clinical improvement, The vast majority of patients are followed up with supportive measures and physiotherapy [6]. 
As previously reported many of these cases are still described as fatal and the prognosis of the surviving patients is variable, depending on the level and extent of the injury [6].

\section{Conclusion}

Fibrocartilaginous embolism of the spinal cord is a rare cause of spinal cord ischemia $[1,2,5]$. It is usually reported as fatal [1-4], but this is questionable since it is most likely underdiagnosed $[1,3]$.

This clinical syndrome should be included in the differential diagnosis of patients with pain associated with sudden neurologic deficit preceded by trauma or physical effort. Among imaging tests, MRI is considered the method of choice for its diagnosis.

\section{References}

1. Tosi L, Rigoli G, Beltramello (1996) Fibrocartilaginous embolism of the spinal cord: a clinical and pathogenetic reconsideration. Neurol Neurosurg Psychiatry 60: 55-60.

2. Mateen FJ, Monrad PA, Hunderfund ANL, Robertson EC, Sorenson EJ (2011) Clinically Suspected fibrocartilaginous embolism: clinical characteristics, treatments, and outcomes. European Journal of Neurology 18: 218-225.

3. Han JJ, Massagli TL, Jaffe KM (2004) Fibrocartilaginous embolism -an uncommon cause of spinal cord infarction: a case report and review of the literature. Arch Phys Med Rehabil 85: 153-157.
4. Novy J, Carruzzo A, Maeder P, Bogousslavsky J (2006) Spinal Cord Ischemia Clinical and Imaging Patterns, Pathogenesis, and Outcomes in 27 Patients. Arch Neurol 63: 1113-1120.

5. Roshal D, Gutierrez C, Brock D, Kremens D (2010) Pearls and Oysters: Fibrocartilaginous embolism myelopathy. Neurology 74 : 21-23.

6. Bansal S, Brown W, Dayal A, Carpenter JL (2014) Posterior spinal cord infarction due to fibrocartilaginous embolization in a 16year-old athlete. Pediatrics 134.

7. Naiman JL, Donohue WL, Prichard JS (1961) Fatal nucleus pulposus embolism of spinal cord after trauma. Neurology 11: 83-87.

8. Kurunlahti M, Kerttula L, Jauhiainen J, Karppinen J, Tervonen O (2001) Correlation of Diffusion in Lumbar Intervertebral Disks with Occlusion of Lumbar Arteries: A Study in Adult Volunteer. RSNA 221: 779-786.

9. Vargas MI, Gariani J, Sztajzel R, Barnaure NI, Delattre BM, et al. (2014) Spinal cord ischemia: Practical imaging tips, pearls, and pitfalls. AJNR 1-6.

10. Barrera SS, Barca BA, Montoto MA, Ferreiro Velasco ME, Cidoncha DM, et al. (2001) Spinal cord infarction: prognosis and recovery in a series of 36 patients. Spinal Cord 39: 520-525.

11. Masson C, Pruvo JP, Meder JF, Cordonnier C, Touzé E, et al. (2004) Spinal cord infarction: clinical and magnetic resonance imaging findings and short term outcome. J Neurol Neurosurg Psychiatry 75: 1431-1435. 